\title{
Important Effect of Defect Parameters on the Characteristics of Thue-Morse Photonic Crystal Filters
}

\author{
Hamed Alipour-Banaei, ${ }^{1}$ Farhad Mehdizadeh, ${ }^{2}$ and Mahdi Hassangholizadeh-Kashtiban ${ }^{2}$ \\ ${ }^{1}$ Electrical Department, Faculty of Engineering, Tabriz Branch, Islamic Azad University, Tabriz, Iran \\ ${ }^{2}$ Electrical Department, Faculty of Engineering, Ahar Branch, Islamic Azad University, Ahar, Iran \\ Correspondence should be addressed to Hamed Alipour-Banaei; alipour@iaut.ac.ir
}

Received 24 November 2012; Revised 22 February 2013; Accepted 24 February 2013

Academic Editor: Jung Huang

Copyright (c) 2013 Hamed Alipour-Banaei et al. This is an open access article distributed under the Creative Commons Attribution License, which permits unrestricted use, distribution, and reproduction in any medium, provided the original work is properly cited.

Design and characterization of optical filters based on photonic crystal Thue-Morse structures are theoretically examined using transfer matrix method. It is shown that by introducing defect layer in the original structure of the proposed filter, main characteristics of it are changed. The main advantage of this defect in Thue-Morse structure is its capability for DWDM communication applications. In other words, achievement of DWDM filter through the Thue-Morse photonic crystal structure is much easier. The desired wavelengths can be achieved by changing the defect parameter. High efficiency of the proposed filter is one of its benefits. The transmission efficiency of this structure is about $96 \%$ and the quality factor is more than 77000 .

\section{Introduction}

The advent of Wavelength Division Multiplexing (WDM) and Dense Wavelength Division Multiplexing (DWDM) technologies is an important step toward realizing all optical communication network $[1,2]$. By use of these technologies one can optimize the capacity of optical fiber by launching multiple $(4,8$, or more) optical channels at different wavelengths into one single fiber.

Optical filters are crucial devises for WDM and DWDM systems $[3,4]$. Besides omitting the noise from channel information, optical filters are used for separating the undesired WDM and DWDM channels from desired channels. Other crucial applications of filters are in demultiplexing multiple channels in WDM and DWDM systems [5-7]. Since the discovery of photonic crystal in 1987 [8, 9], designing compact and highly selective optical filters has become possible. Thanks to these artificial periodic structures we can control the flow of light in ultrasmall scales [10]. Due to the simplicity of design and fabrication, 1D PhC based filters are the mass popular devices realized based on $\mathrm{PhC}$. The key feature of PhCs is a wavelength region in which no optical wave is allowed to propagate inside the crystal; this feature is called Photonic Band Gap (PBG) [11]. We can use the PBG property of $1 \mathrm{D}$ PhC to design optical reflector [12] and band reject filters [13]. Adding a defect layer to a $1 \mathrm{D}$ PhC creates a narrow band transmission filter [14]. Also if we replace the defect layer by a photonic quantum well [15] we will have a multichannel filter. By replacing dielectric layers by super conducting $\mathrm{PhC}$ we can realize a multichannel filter without using defect layer or quantum well [16]. In 1984 Shechtman [17] proposed a new class of crystals called quasi-periodic and aperiodic crystals. Fibonacci, Thue-Morse, and Rodin-Shapiro structures are some example of nonperiodic photonic crystals. It has been shown that in Thue-Morse (ThM) structures increasing the order of system results in exponential increase in the density of resonance peaks [18]. Studying the propagation of light wave and localization properties of light in ThM multilayers showed that around the midgap frequencies the transmission is sensitive to optical thickness modulation [19]. Another advantage of ThM structure over ordinary periodic 1D PhCs has been proposed by Agarwal et al. [20] they found on enhancement in the number of PBG with a blue shift in reflectance peaks of ThM structure. Intrinsic asymmetry 


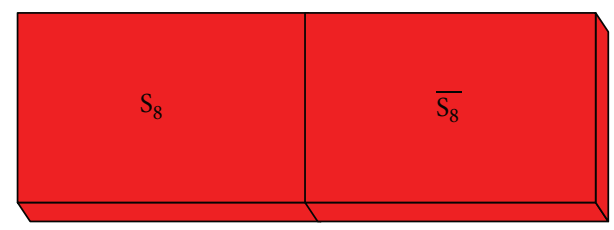

FIGURE 1: The schematic diagram of $\mathrm{S}_{9}$ ThM structure without defect layer.

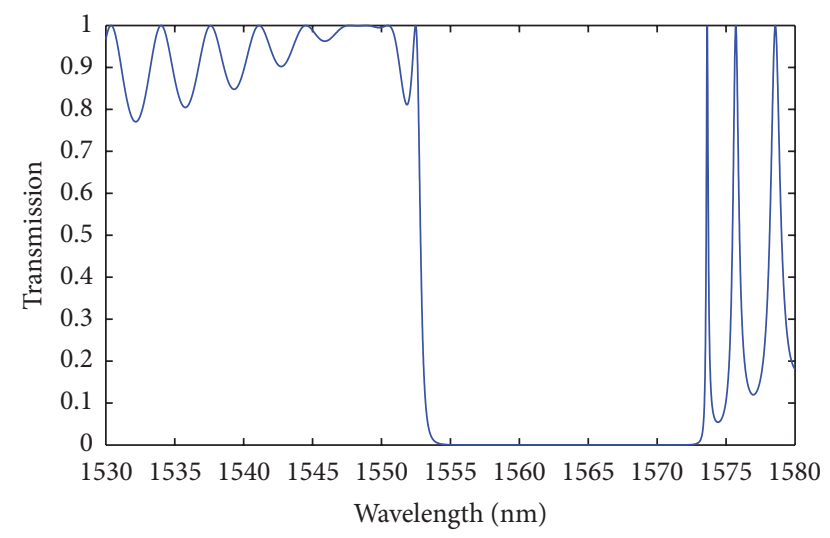

FIgURE 2: The transmission spectrum of $S_{9}$ structure shown in Figure 1.

of 1D ThM PhC in odd generation results in bistability, thresholds, that are structure to propagation direction so these structure can be used for designing all optical diode [21]. In Multicomponent Generalized TM (MCGTM) supper lattices optical transmission has an interesting pseudoconstant characteristic at the central wavelength due to contra-set structure of these structures which can be used for designing complex optical devices [22]. A tunable filter based on TMPhC composed of single negative materials is proposed which is not sensitive to incident angle and polarization of light. It has been shown that bandwidth and frequency of this filter can be changed by adjusting the layer thicknesses and plasma frequency [23]. In 1D TM PhC composed of negative refractive index and positive refractive index materials there is an enlarged Omnidirectional Reflection Band (ORB) and the width and location of ORB are independent of TM order; the lower and higher edge of ORB depends only on TE and TM polarizations, respectively [24]. The most crucial advantages of ThM based PhC filters over periodic PhC filters are high transmission efficiency, small bandwidth, and high quality factor [25]. Other works have been done on ThM structures which have been devoted to the transmission and PBG properties of these structures [26-29].

In this work we are going to propose a new method for designing ThM based $1 \mathrm{D}$ PhC filter. In our work we introduced a defect layer inside the 9th order $1 \mathrm{D}$ ThMPhC structure. The significant characteristics of our filter are its very high transmission efficiency, very low bandwidth, and very high quality factor. The transmission efficiency of this structure is about $96 \%$ and the quality factor is more than 77000 .

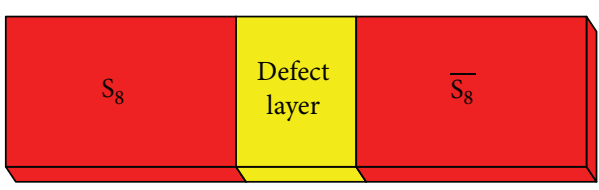

Figure 3: The final sketch of our proposed filter.

The rest of the paper is organized as follows. In Section 2 the design procedure of the filter has been proposed. In Section 3 we discussed the simulation process and the results and finally in Section 4 we concluded our work.

\section{Filter Design}

In this paper, we are going to propose a tunable filter based on defective 1D ThM structure. So first of all we are going to introduce ThM structure briefly.

2.1. Thue-Morse Structure. ThM is a binary sequence defined as follows:

$$
\mathrm{S}_{n}=\mathrm{S}_{n-1} \mathrm{~S}_{n-1}^{*} .
$$

Some examples of ThM sequence are $S_{1}=10, S_{2}=1001$, $S_{3}=10010110$, and so on.

Now if one substitutes 1's and 0's with A and B layers, where $\mathrm{A}$ and $\mathrm{B}$ are dielectric layers with different refractive indices and thicknesses $\left(n_{\mathrm{A}}\right.$ and $n_{\mathrm{B}}$ are the refractive index of $\mathrm{A}$ and $\mathrm{B}$, resp., and $h_{\mathrm{A}}$ and $h_{\mathrm{B}}$ are the thicknesses of $\mathrm{A}$ and $\mathrm{B}$, resp.).

So some example of ThM structure will be as follows: $\mathrm{S}_{0}=$ $\mathrm{A}, \mathrm{S}_{1}=\mathrm{AB}, \mathrm{S}_{2}=\mathrm{ABBA}, \mathrm{S}_{3}=\mathrm{ABBABAAB}$, and so on.

In order to design proposed filter we used 9 th order ThM structure; according to (1) 9th order ThM structure is as follows:

$$
\mathrm{S}_{9}=\mathrm{S}_{8} \mathrm{~S}_{8}^{*}
$$

so

$$
\mathrm{S}_{9}=\text { ABBABAABBAABABBA } \ldots .
$$

In this structure the refractive index of $A$ and $B$ layers (e.g., $n_{\mathrm{A}}$ and $n_{\mathrm{B}}$ ) are 3.17 and 2 , respectively, and the thicknesses of these layers are $h_{\mathrm{A}}=235 \mathrm{~nm}$ and $h_{\mathrm{B}}=$ $798 \mathrm{~nm}$. Currently the best solution for studying the optical properties of 1D multilayer structure like 1D PhCs is Transfer Matrix Method (TMM). The fundamental structure used for designing our proposed filter is shown in Figure 1 and the transmission spectra of this structure with aforementioned values for $n_{\mathrm{A}}, n_{\mathrm{B}}, h_{\mathrm{A}}$, and $h_{\mathrm{B}}$ are plotted in Figure 2. Figure 2 shows that we have a PBG in $1550 \mathrm{~nm}<\lambda<1570 \mathrm{~nm}$ range. This PBG range is suitable for optical communication applications and WDM and DWDM technologies.

The next step in designing of the proposed filter is creating a transmission channel in the PBG region. As far as we know in ordinary periodic 1D PhCs, this will be done by introducing a defect layer into the structure. So in our filter 


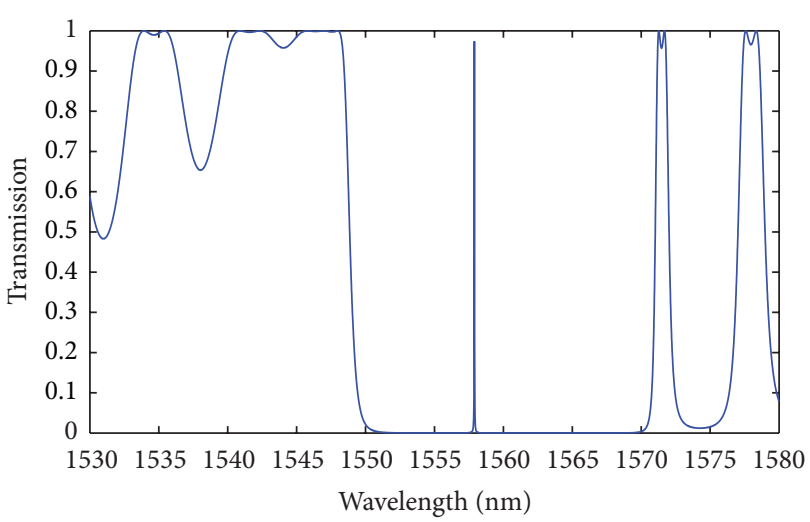

(a)

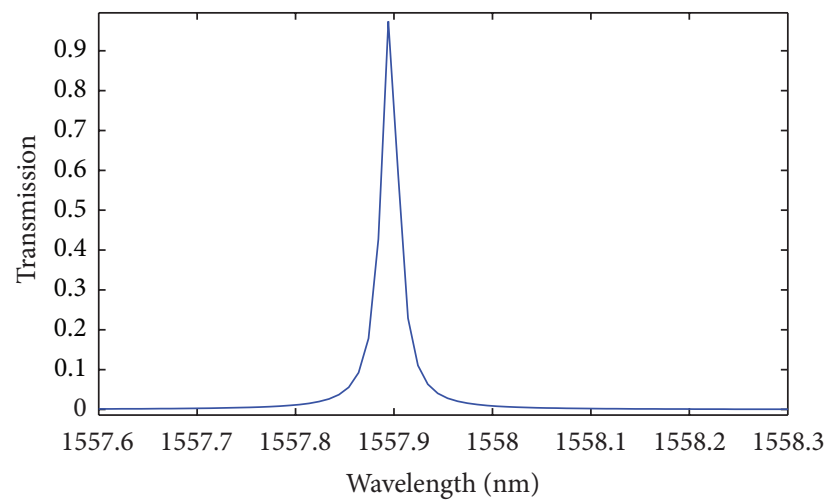

(b)

FIgURE 4: The output spectrum of our proposed filter: (a) normal view and (b) zoomed in view.

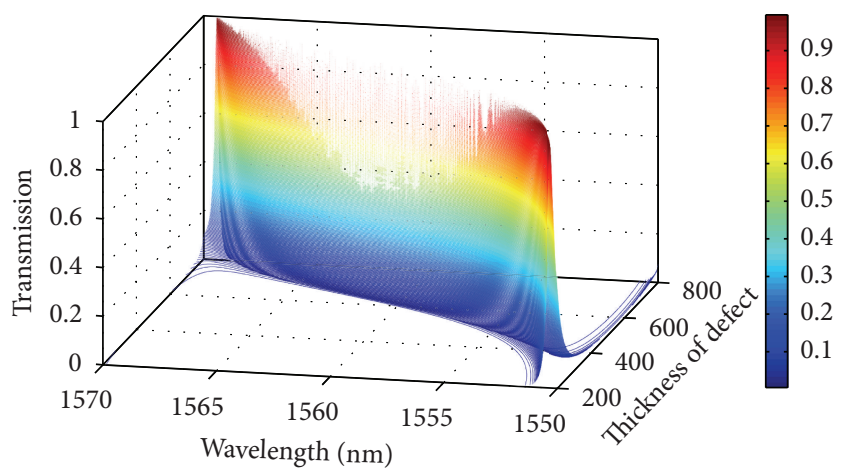

FIGURE 5: The 3D plot of the output spectra of the filter for different values of $h_{\mathrm{D}}$.

we introduce a defect layer in to the ThM structure, so the final sketch of our filter will be like Figure 3 .

We call the refractive index and thickness of defect layer $n_{\mathrm{D}}$ and $h_{\mathrm{D}}$, respectively. In this filter $n_{\mathrm{D}}$ and $h_{\mathrm{D}}$ are 1 and $554 \mathrm{~nm}$, respectively. The transmission spectrum for the defective structure (final sketch of filter) is shown in Figure 4.

As we observe from Figure 4 we have a transmission channel at $\lambda=1557.9 \mathrm{~nm}$. This proves that the effect of defect layer in ordinary $1 \mathrm{D} \mathrm{PhC}$ is true for ThM based multilayers, too. The transmission efficiency of the proposed structure is about $96 \%$.

\section{Simulation and Results}

As we mentioned in the previous section, we use defective mode of 9th order ThM structure for designing our filter; the final structure of our filter will be as follows: $\mathrm{F}=\mathrm{S}_{8} \mathrm{DS}_{8}^{*}$ which is shown schematically in Figure 3. After finalizing the design procedure of filter, we are going to investigate the effect of $h_{\mathrm{D}}$-the thickness of the defect layer-on the filtering behavior of the proposed structure. The transmission spectra of the proposed filter for different values of $h_{\mathrm{D}}$ are shown in Figures 5, 6, and 7. Figure 5 is a 3D plot of the output spectrum of the filter, which shows the transmission efficiency and output wavelength of the proposed filter for various values of $h_{\mathrm{D}}$. The obvious point we obtain from Figure 5 is that our filter is tunable. This figure shows that by changing the thickness of the defect layer from $400 \mathrm{~nm}$ to $800 \mathrm{~nm}$ the output wavelength will change from $1550 \mathrm{~nm}$ to $1570 \mathrm{~nm}$. It means that by increasing the thickness of the defect layer the output wavelength will shift towards upper wavelengths.

Figure 6 is the top view of Figure 5. In this figure, the vertical and horizontal axes are representative of wavelength and the thickness of the defect layer and the transmission efficiency is shown by the color scale, which is shown next to the figure. The white region in Figure 6(a) is the PBG region and the multi-color curve inside the white region is the defect mode that has been created by inserting a defect layer into the ThM structure. We observe that by increasing the thickness of the defect layer $\left(h_{\mathrm{D}}\right)$ the multi-color curve shifts towards higher wavelengths. Figure 6(b) is the zoomed in form of Figure 6(a) in which we zoomed in around the central wavelength of one channel according to the color scale and we see that the peak of the output spectrum for $h_{\mathrm{D}}=554.6 \mathrm{~nm}$ and $\lambda=1557.96 \mathrm{~nm}$ whose transmission efficiency is about $96 \%$.

We also observe that the bandwidth of the transmission channel is very small and the output spectrum is very sharp so the quality factor of our proposed filter is very high.

The bandwidth is less than $0.02 \mathrm{~nm}$ and the quality factor is more than 70000 . Finally the output wavelength of the filter for different values of $h_{\mathrm{D}}$ is shown in Figure 7. This figure shows the high transmission efficiency and narrow band properties of the proposed filter.

\section{Conclusion}

We have proposed the design of a compact optical filter in a Thue-Morse structure with photonic crystals. Since the filter does not involve either additional materials or other complexities, it might realize the tough fabrication 


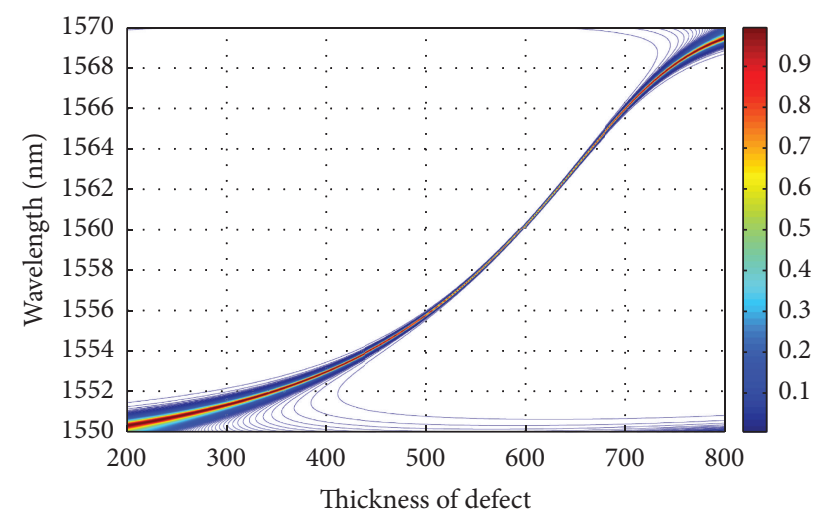

(a)

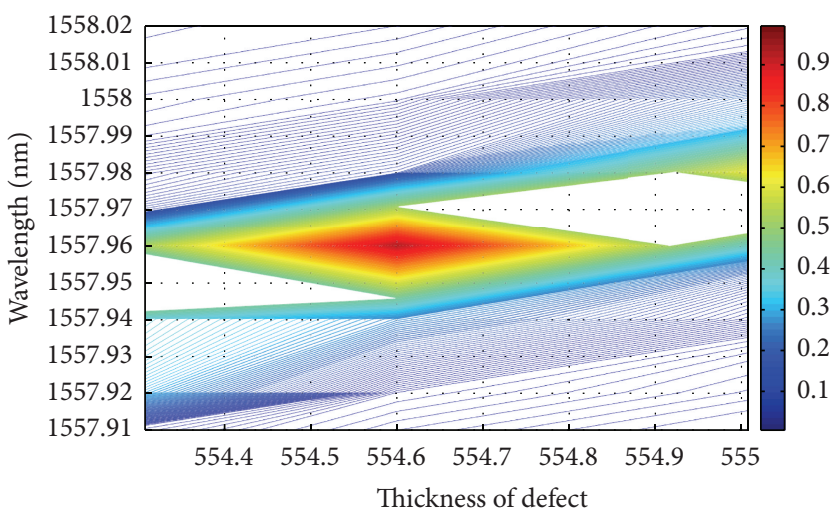

(b)

FIgURE 6: The top view of Figure 5. The variation of output wavelength versus $h_{\mathrm{D}}$ : (a) normal view, (b) zoomed in view.

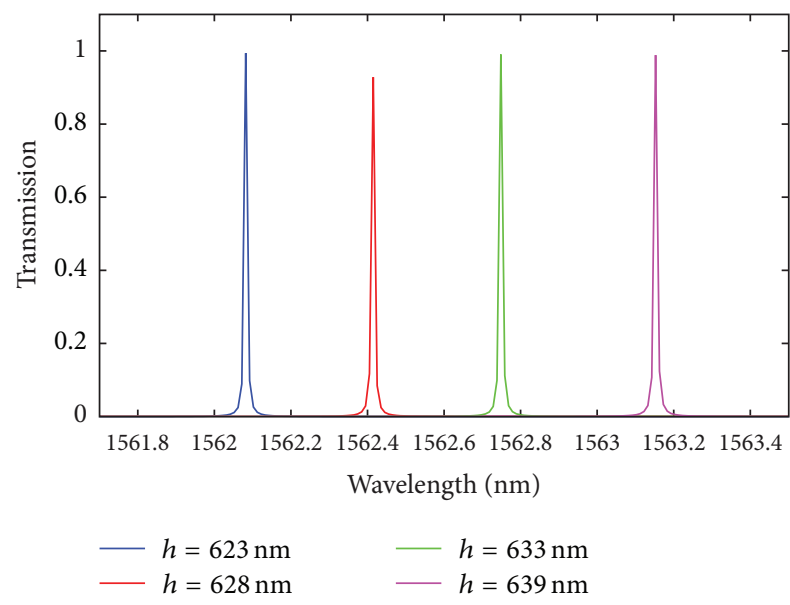

FIGURE 7: The output spectra of our proposed filter for different values of $h_{\mathrm{D}}$.

requirements. By introducing defect layer between the ThueMorse structure layers, dependency of the proposed filter to the defect layer is shown. The efficiency of the designed filter is very good and is about $96 \%$. The proposed filter is suitable for WDM and DWDM communication applications due to its narrow band transmission spectra.

\section{References}

[1] S. G. Mun, J. H. Moon, H. K. Lee, J. Y. Kim, and C. H. Lee, "A WDM-PON with a $40 \mathrm{~Gb} / \mathrm{s}(32 \times 1.25 \mathrm{~Gb} / \mathrm{s})$ capacity based on wavelength-locked Fabry-Perot laser diodes," Optics Express, vol. 16, no. 15, pp. 11361-11368, 2008.

[2] I. Gasulla and J. Capmany, “Tb/s.km Multimode fiber link combining WDM transmission and low-linewidth lasers," Optics Express, vol. 16, pp. 8033-8038, 2008.

[3] Z. Xu, J. Wang, Q. He, L. Cao, P. Su, and G. Jin, "Optical filter based on contra-directional waveguide coupling in a $2 \mathrm{D}$ photonic crystal with square lattice of dielectric rods," Optics Express, vol. 13, no. 15, pp. 5608-5613, 2005.
[4] H. Ghafoori-Fard, M. J. Moghimi, and A. Rostami, "Linear and nonlinear superimposed bragg grating: a novel proposal for alloptical multi-wavelength filtering and switching," Progress in Electromagnetics Research, vol. 77, pp. 243-266, 2007.

[5] X. Sun, P. Gu, M. Li, X. Liu, D. Wang, and J. Zhang, "Tunable spatial demultiplexer based on the Fabry-Perot filter," Optics Express, vol. 14, pp. 8470-8475, 2006.

[6] H. Takahashi, S. Suzuki, K. Kato, and I. Nishi, "Arrayedwaveguide grating for wavelength division multi/demultiplexer with nanometre resolution," Electronics Letters, vol. 26, no. 2, pp. 87-88, 1990.

[7] M. Gerken and D. A. B. Miller, "Wavelength demultiplexer using the spatial dispersion of multilayer thin-film structures," IEEE Photonics Technology Letters, vol. 15, no. 8, pp. 1097-1099, 2003.

[8] E. Yablonovitch, "Inhibited spontaneous emission in solid-state physics and electronics," Physical Review Letters, vol. 58, no. 20, pp. 2059-2062, 1987.

[9] S. John, "Strong localization of photons in certain disordered dielectric superlattices," Physical Review Letters, vol. 58, no. 23, pp. 2486-2489, 1987.

[10] S. J. McNab, N. Moll, and Y. A. Vlasov, "Ultra-low loss photonic integrated circuit with membrane-type photonic crystal waveguides," Optics Express, vol. 11, no. 22, pp. 2927-2939, 2003.

[11] K. Sakoda, Optical Properties of Photonic Crystals, Springer, Berlin, Germany, 2001.

[12] F. Mehdizadeh, H. Alipour-Banaei, and Z. Daie-Kuzekanani, "All optical multi reflection structure based on one dimensional photonic crystals for WDM communication systems," Optoelectronics and Advanced Materials-Rapid Communications, vol. 6, pp. 527-531, 2012.

[13] H. Alipour-Banaei and F. Mehdizadeh, "A proposal for antiuvb filter based on one-dimensional photonic crystal structure," Digest Journal of Nanomaterials and Biostructures, vol. 7, pp. 361-367, 2012.

[14] C. J. Wu and Z. H. Wang, "Properties of defect modes in onedimensional photonic crystals," Progress in Electromagnetics Research, vol. 103, pp. 169-184, 2010.

[15] F. Qiao, C. Zhang, J. Wan, and J. Zi, "Photonic quantum-well structures: multiple channeled filtering phenomena," Applied Physics Letters, vol. 77, pp. 3698-3701, 2000. 
[16] W. H. Lin, C. J. Wu, T. J. Yang, and S. J. Chang, "Terahertz multichanneled filter in a superconducting photonic crystal," Optics Express, vol. 18, no. 26, pp. 27155-27166, 2010.

[17] D. Shechtman, I. Blech, D. Gratias, and J. W. Cahn, "Metallic phase with long-range orientational order and no translational symmetry," Physical Review Letters, vol. 53, no. 20, pp. 1951-1953, 1984.

[18] W. J. Hsueh, S. J. Wun, Z. J. Lin, and Y. H. Cheng, "Features of the perfect transmission in Thue-Morse dielectric multilayers," Journal of the Optical Society of America B, vol. 28, pp. 25842591, 2011.

[19] N. Liu, "Propagation of light waves in Thue-Morse dielectric multilayers," Physical Review B, vol. 55, pp. 3543-3547, 1997.

[20] V. Agarwal, J. A. Soto-Urueta, D. Becerra, and M. E. MoraRamos, "Light propagation in polytype Thue-Morse structures made of porous silicon," Photonics and Nanostructures, vol. 3, no. 2-3, pp. 155-161, 2005.

[21] V. V. Grigoriev and F. Biancalana, "Bistability and stationary gap solitons in quasiperiodic photonic crystals based on ThueMorse sequence," Photonics and Nanostructures, vol. 8, no. 4, pp. 285-290, 2010.

[22] G. Zhang, X. Yang, Y. Li, and H. Song, "Optical transmission through multi-component generalized ThueMorse superlattices," Physica B, vol. 405, no. 17, pp. 3605-3610, 2010.

[23] X. H. Deng, J. R. Yuan, W. Q. Hong, and H. Ouyang, "Tunable filters based on Thue-Morse quasicrystals composed of singlenegative materials," Physics Procedia, vol. 22, pp. 360-365, 2011.

[24] O. Y. Hong and X. H. Deng, "Direction-independent band gaps extension based on Thue-Morse photonic heterostructures containing negative-index materials," Materials Science Forum, vol. 675, pp. 1077-1080, 2011.

[25] S. Zirak-Gharamaleki, "Narrowband optical filter design for DWDM communication applications based on Generalized Aperiodic Thue-Morse structures," Optics Communications, vol. 284, no. 2, pp. 579-584, 2011.

[26] M. S. Vasconcelos and E. L. Albuquerque, "Transmission fingerprints in quasiperiodic dielectric multilayers," Physical Review B, vol. 59, no. 17, pp. 11128-11131, 1999.

[27] L. Dal Negro, M. Stolfi, Y. Yi et al., "Photon band gap properties and omnidirectional reflectance in $\mathrm{Si} / \mathrm{SiO}_{2}$ Thue-Morse quasicrystals," Applied Physics Letters, vol. 84, pp. 5186-5188, 2004.

[28] X. Jiang, Y. Zhang, S. Feng, K. C. Huang, Y. Yi, and J. D. Joannopoulos, "Photonic band gaps and localization in the Thue-Morse structures," Applied Physics Letters, vol. 86, Article ID 201110, 3 pages, 2005.

[29] L. Dal Negro, J. H. Yi, V. Nguyen, Y. Yi, J. Michel, and L. C. Kimerling, "Spectrally enhanced light emission from aperiodic photonic structures," Applied Physics Letters, vol. 86, Article ID 261905, 3 pages, 2005. 

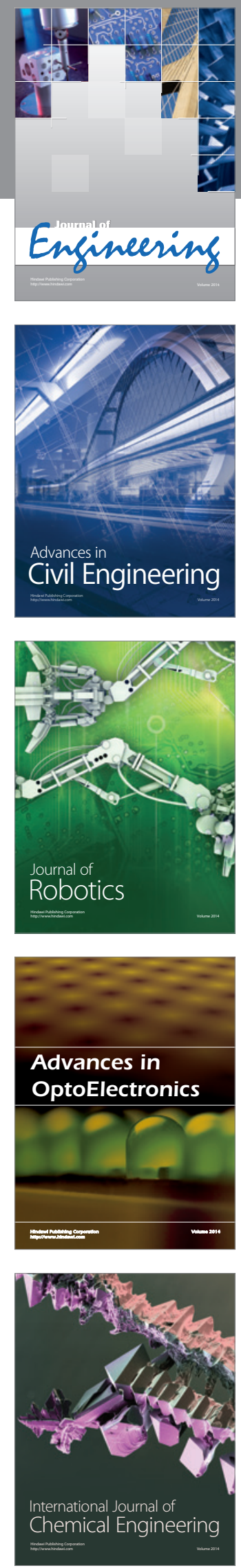

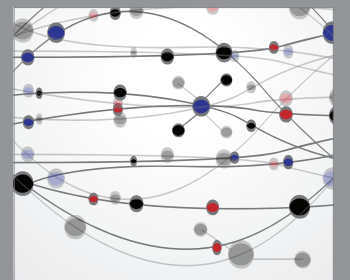

The Scientific World Journal
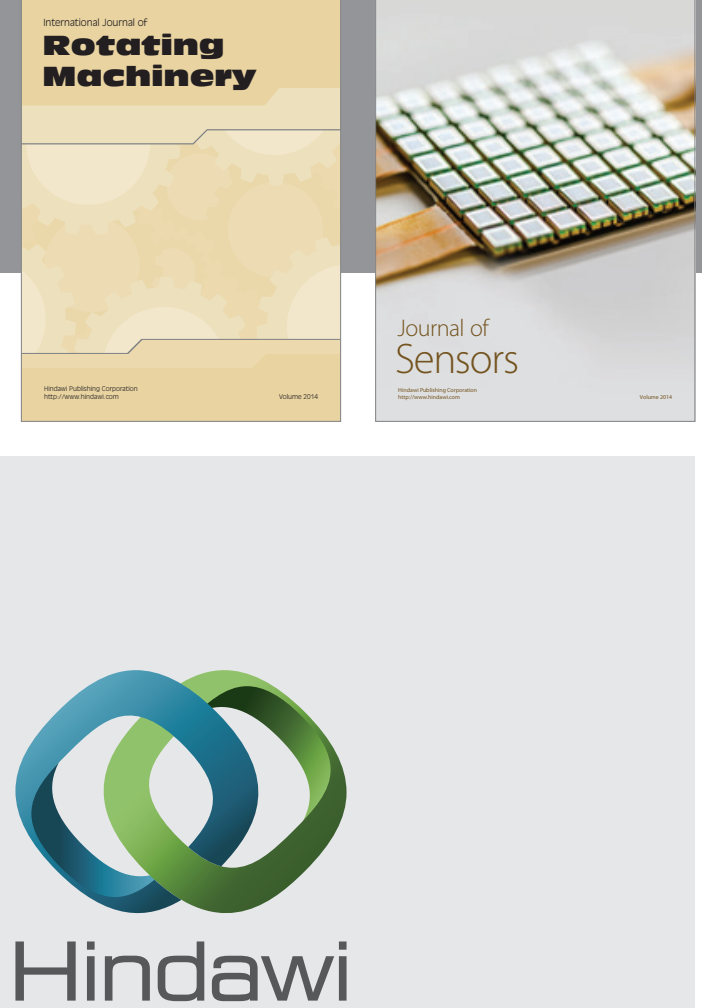

Submit your manuscripts at http://www.hindawi.com
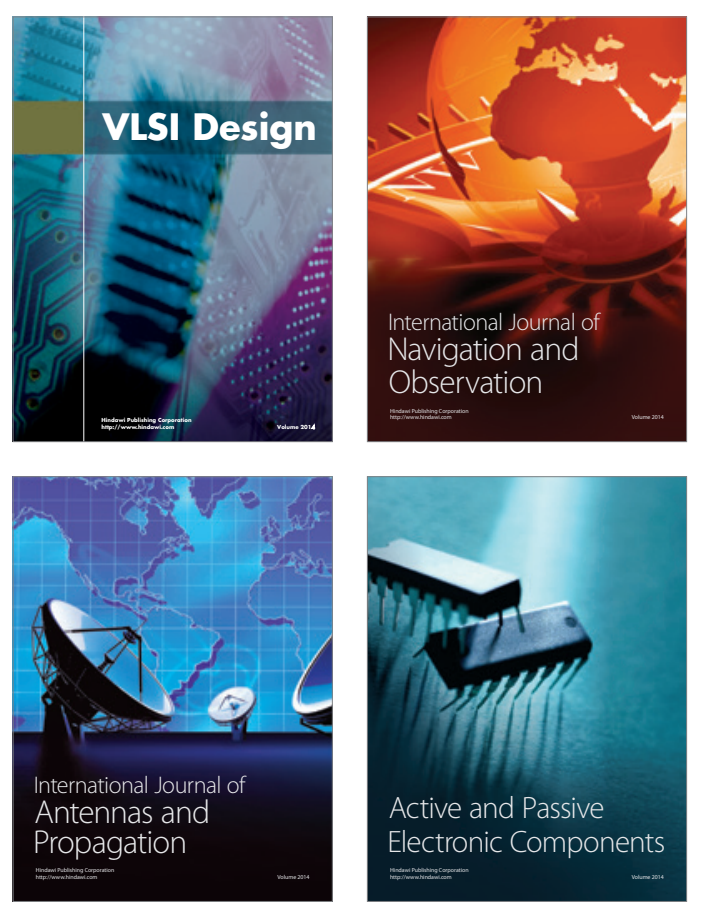
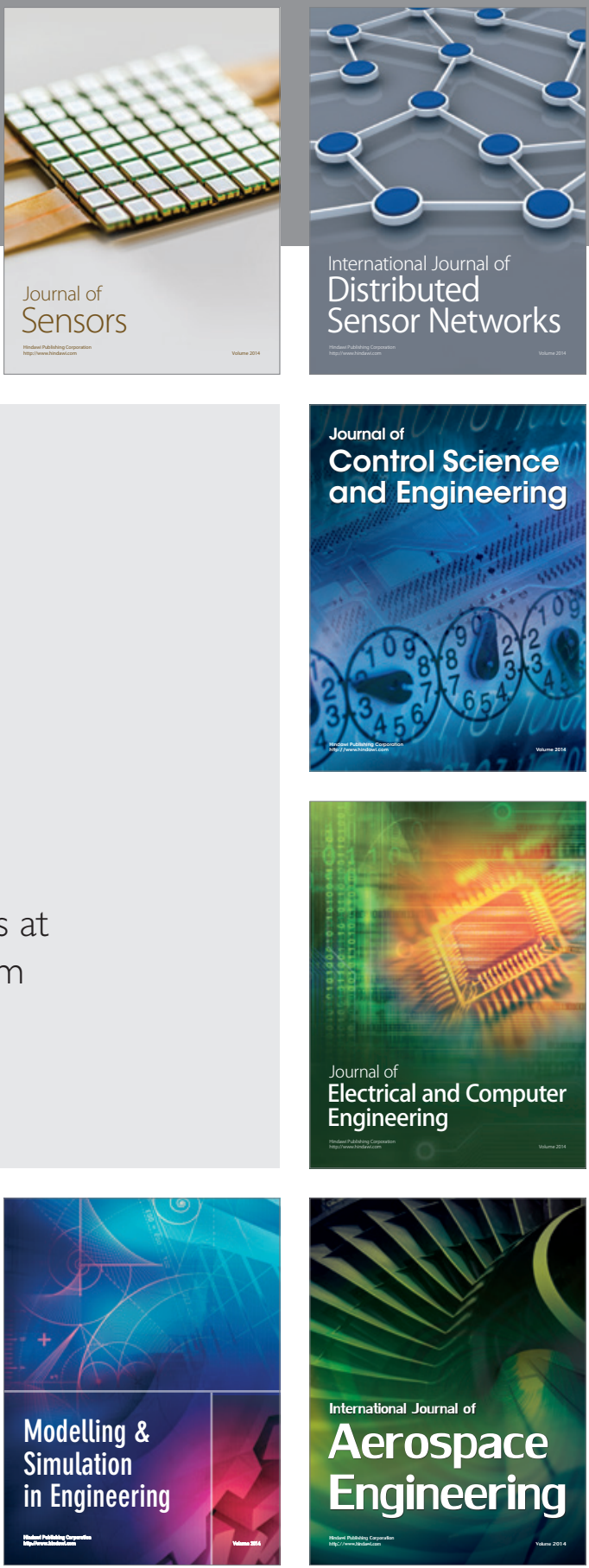

Journal of

Control Science

and Engineering
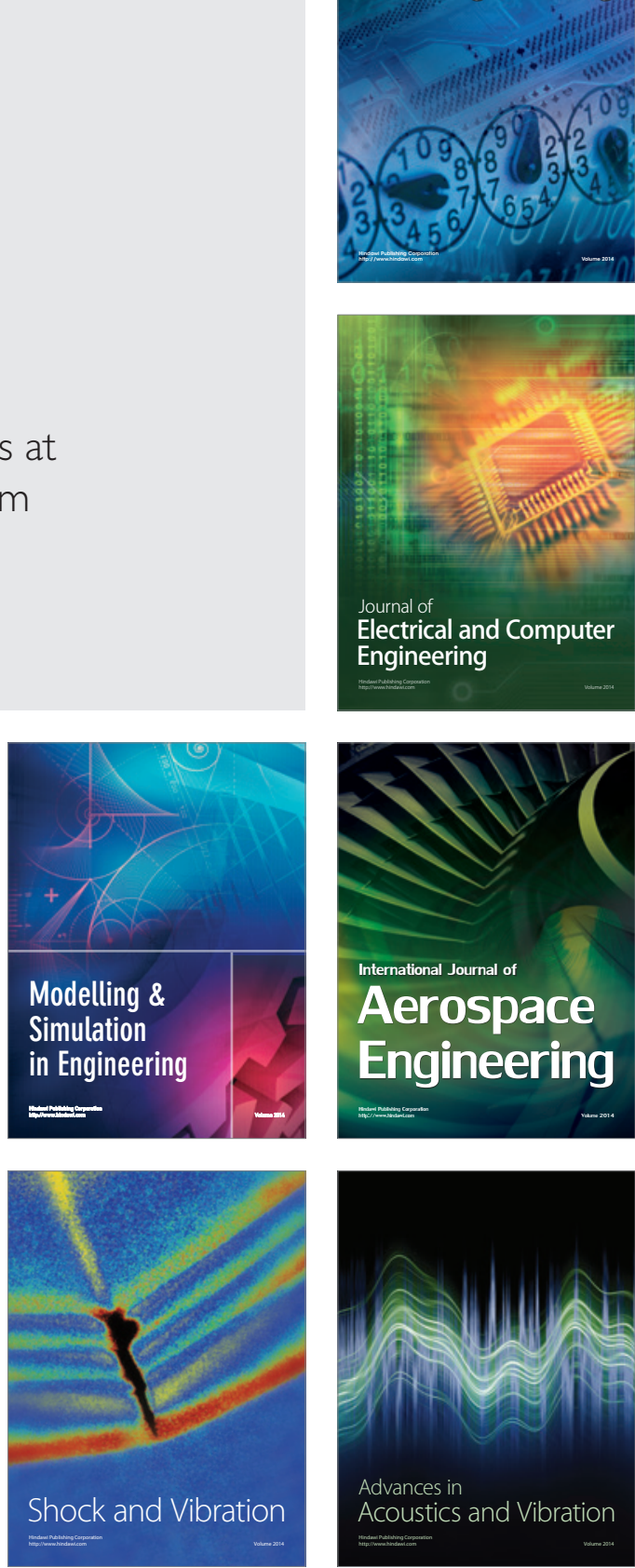